\title{
Hepatocellular carcinoma with complete response to the immunotherapy: the oncologist's dilemma
}

\author{
Mansour Al-Moundhri ${ }^{1}$, Antonio Cubisino ${ }^{2}$, Fabrizio Panaro $^{2,3,4}$ \\ ${ }^{1}$ Medical Oncology Department, Sultan Qaboos Comprehensive Cancer Center, Sultan Qaboos University, Muscat, Oman; ${ }^{2}$ Department of Surgery, \\ University of Illinois at Chicago, Chicago, IL, USA; ${ }^{3}$ Department of Surgery, Montpellier University Hospital, Montpellier, France; ${ }^{4}$ Surgical \\ Oncology Department, Sultan Qaboos Comprehensive Cancer Center, Sultan Qaboos University, Muscat, Oman \\ Correspondence to: Prof. Fabrizio Panaro, MD, PhD. Department of Surgery/Division of HBP Surgery and Liver Transplantation, Montpellier \\ University Hospital-School of Medicine, 34000 Montpellier, France. Email: f-panaro@chu-montpellier.fr.
}

Submitted Nov 29, 2021. Accepted for publication Dec 09, 2021.

doi: 10.21037/hbsn-21-499

View this article at: https://dx.doi.org/10.21037/hbsn-21-499

Hepatocellular carcinoma (HCC) is the seventh most common cancer and the fourth leading cause of cancerrelated death worldwide (1). During the last decade, many efforts have been made to improve patient survival by conducting clinical trials investigating local and systemic treatment options for patients with unresectable tumors. However, despite significant research efforts, only few treatment approaches have been validated for HCC.

Immunotherapy is a novel treatment approach representing an effective, promising and safe option against HCC.

Recently, six systemic therapies have been approved based on phase III trials (atezolizumab plus bevacizumab, sorafenib, lenvatinib, regorafenib, cabozantinib and ramucirumab) and three additional therapies have obtained accelerated Food and Drug Administration (FDA) approval owing to evidence of efficacy (2).

The combination of atezolizumab and bevacizumab is the current standard of care for patients with locally advanced or metastatic HCC. The approval of the combination was based on IMbrave 150 study which showed clear superiority over Sorafenib in terms of OS [hazard ratio (HR) 0.66; $95 \%$ CI: 0.92-0.85; $\mathrm{P}=0.0006$ ] and median PFS (HR 0.59; $95 \%$ CI: $0.47-0.76$; $\mathrm{P}<0.0001)$. Moreover, there was tripling of response from $11 \%$ for Sorafenib to $30 \%$ for atezolizumab and bevacizumab with $7.7 \%$ achieving complete response (3).

HCC is an aggressive disease with poor prognosis with median overall survival, 10.7-13.4 months (Sharp and IMbrave study) when treated with Sorafenib $(3,4)$. However, the use of atezolizumab and bevacizumab has resulted in significant improvement in median OS to 19.2 months and duration of response of 18.1 and 16.3 months on mRECIST scale (3). However, the increased use of immunotherapy has demonstrated a wide variety of imaging features and patterns of disease that cannot be adequately captured by traditional response criteria, such as the World Health Organization (WHO) criteria and Response Evaluation Criteria in Solid Tumors (RECIST), which have been used primarily with cytotoxic chemotherapies. In response to these observations, several novel response criteria have been developed to evaluate patients who receive immunotherapy.

Thus, new criteria, such as immune-related response criteria (irRC), immune-related RECIST (irRECIST), and immune-RECIST (iRECIST), have been developed to assist clinicians in distinguishing between patients who are and are not responding to a particular treatment (5) (Table 1).

Positron emission tomography (PET) using $18 \mathrm{~F}$-fluorodeoxyglucose $\left({ }^{18} \mathrm{~F}-\mathrm{FDG}\right)$ is widely used for assessing a variety of malignancies, has poor sensitivity in the evaluation of HCC.

In this effort, novel PET radiotracers (11C-acetate and $11 \mathrm{C}$-choline) are currently being assessed. In addition, investigational use of magnetic resonance (MR) radiomics for depicting apoptosis and cytolysis to monitor cancer response also has been reported (6). Other radiology investigations are in various stages of development. In this setting, molecular imaging with immuno-positron emission tomography (immuno-PET) provides a noninvasive functional biomarker of tumor response for patients on immunotherapy (5).

Therefore, a clinical management issue arises regarding 
Table 1 New immune-related response criteria

\begin{tabular}{lllll}
\hline Response criteria & irRC & irRECIST & iRECIST & RECIST 1.1 \\
\hline CR & $\begin{array}{l}\text { Disappearance of all } \\
\text { lesions }\end{array}$ & $\begin{array}{l}\text { Disappearance of all } \\
\text { lesions }\end{array}$ & $\begin{array}{l}\text { Disappearance of all } \\
\text { lesions }\end{array}$ & Disappearance of all lesions \\
PR & $\begin{array}{l}\geq 50 \% \text { decrease from } \\
\text { baseline }\end{array}$ & $\begin{array}{l}\geq 30 \% \text { decrease from } \\
\text { baseline }\end{array}$ & $\begin{array}{l}\geq 30 \% \text { decrease from } \\
\text { baseline }\end{array}$ & $\begin{array}{l}>30 \% \text { decrease from } \\
\text { baseline }\end{array}$ \\
SD & Neither CR nor PD is met & Neither CR nor PD is met & Neither CR nor PD is met & Neither CR nor PD is met \\
PD & $\geq 25 \%$ increase & $\begin{array}{l}\geq 20 \% \text { increase (minimum } \\
\text { of } 5 \mathrm{~mm} \text { ) }\end{array}$ & of $5 \mathrm{~mm}$ ) & $>20 \%$ increase (minimum \\
& & of $5 \mathrm{~mm}$ )
\end{tabular}

irRC, immune-related response criteria; irRECIST, immune-related RECIST; iRECIST, immune-RECIST; RECIST, Response Evaluation Criteria in Solid Tumors; CR, complete response; PR, partial response; SD, stable disease; PD, progressive disease.

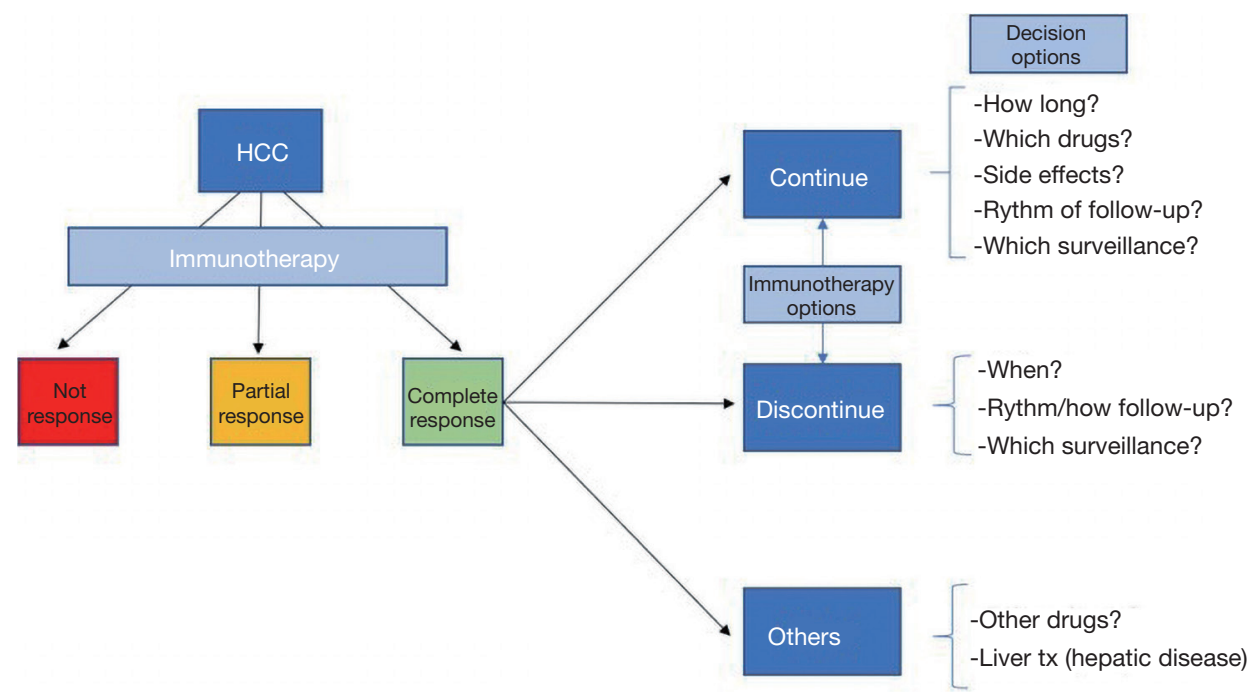

Figure 1 Decision making flow chart. HCC, hepatocellular carcinoma; Liver tx, liver transplantation.

the duration of immunotherapy with PD-L1 inhibitor atezolizumab and bevacizumab in a subset of HCC patients who will achieve durable complete response (CR) and long survival. To answer this question, we need to take in consideration several factors that include design of study, toxicity profile, quality of life on treatment, and costeffectiveness (Figure 1).

The design of IMbrave 150 study continued atezolizumab and bevacizumab combination until disease progression or unacceptable toxicity (3). Therefore, it is seldom that we deviate from study design in order not to compromise obtained treatment outcomes.

The combination of atezolizumab and bevacizumab results in significant toxicity with $38.0 \%$ of patient developing serious toxicity of grade 3 and 4 and grade 5 toxicity occurred in $5.8 \%$ with the discontinuation rate of
$15 \%$ and $49.5 \%$ of patients having modification of dose interruption of treatment (3). The combination treatment was shown to delay deterioration in quality-of-life with median time of 11.2 months with compliance rate up to $93 \%$ from baseline until cycle 17 of treatment and drops to $80 \%$ after that until discontinuation of treatment (3). Therefore, we can surmise that small percentage of treated patient who will complete two years on treatment partly due toxicity of treatment in addition to disease progression.

The balance between efficacy and toxicity of such treatment is crucial to take a good decision. The majority of toxicities are mild to moderate in severity, however, serious and occasionally life-threatening (treatment-related deaths $2 \%$ ) adverse effects are reported in the literature (7). Immune-toxicity resulting from immunotherapy can have a delayed onset compared to chemotherapy, and effective 
management depends on early recognition and prompt intervention.

In this contest, to provide guidance to the oncology community on important concerns for the immunotherapeutic care of HCC, the Society for Immunotherapy of Cancer (SITC) established a multidisciplinary Toxicity Management Working Group to develop a clinical practice guideline to standardize management of toxicity (8).

Based on the European Society of Medical Oncology (ESMO) guidelines for the HCC on immunotherapy treatment are evaluated clinically for signs of liver decompensation and for cancer progression by dynamic CT or MRI every 3 months (III, A) also in case of complete response (9). These guidelines of surveillance can also be transposed for those patients who have stopped immunotherapy due to toxicity.

In terms of cost-effectiveness the combination of atezolizumab and bevacizumab was shown to be of clinical benefit but was not cost-effective compared to sorafenib. The incremental increase in the quality-of-life years per patient was US $\$ 156,210$ and an increment cost effective ratio of 322,500 per quality-adjusted life year (10).

The extrapolation from other cancer models can be considered in the attempt optimal duration of immunotherapy in HCC despite the inherent biological differences between various cancers. For example, in melanoma it has been demonstrated that treatment beyond two years is of no clinical value. On the other hand, treatment less than 18 months even in patients achieving CR may be determined in terms of PFS. Therefore, it is of paramount importance to study the biological and clinical prognostic factors that may best determine the best outcome in each cancer in relation to duration of immunotherapy. Real time data in terms of duration of immunotherapy in HCC in patients achieving CR versus partial response (PR) or stable disease (SD) is highly relevant in particular. Moreover, the study's biological factors that may predict the subset who benefit from immunotherapy is an evolving area of research, for example immune signature has been shown to have a predictive value. Genes from various signaling pathways (telomere maintenance, P53/cell cycle regulation, $\mathrm{Wnt} / \beta$-catenin, AKT/mTOR and MAP kinase) are frequently mutated in HCC (11). Moreover, mutations in members of DNA damage repair (DDR) pathways may affect the efficacy of immunotherapy. Alterations in DDR signaling pathway can lead to genomic instability and increased mutation frequency. Mutations can be used as potential biomarkers for the efficacy of immunotherapy (11).
The availability of pre- and post-treatment tumour samples during immunotherapy is crucial for biomarker research in HCC and to predict the impact of individual agents with different immunomodulatory actions.

In conclusion, the combination of atezolizumab and bevacizumab represent a significant advancement in the treatment of unresectable or metastatic HCC. IMbrave 150 is the only randomized study that have shown superiority offers the combination treatment that was administered until disease progression on unresectable toxicity, and in our opinion, this approach is only evidenced-based valid approach to duration of immunotherapy treatment in HCC. However, it should be noted that the issue of duration of immunotherapy will be faced in small highly selected proportion of HCC patients as large number of patients do not obtain clinical benefit or durable response. This small group of selected patients who have durable duration should be studied for predictive determinants of biological and clinical factors.

\section{Acknowledgments}

Funding: None.

\section{Footnote}

Provenance and Peer Review: This article was commissioned by the editorial office, Hepatobiliary Surgery and Nutrition. The article did not undergo external peer review.

Conflicts of Interest: All authors have completed the ICMJE uniform disclosure form (available at https://hbsn. amegroups.com/article/view/10.21037/hbsn-21-499/coif). FP serves as an unpaid editorial board member of Hepatobiliary Surgery and Nutrition. The other authors have no conflicts of interest to declare.

Ethical Statement: The authors are accountable for all aspects of the work in ensuring that questions related to the accuracy or integrity of any part of the work are appropriately investigated and resolved.

Open Access Statement: This is an Open Access article distributed in accordance with the Creative Commons Attribution-NonCommercial-NoDerivs 4.0 International License (CC BY-NC-ND 4.0), which permits the noncommercial replication and distribution of the article with the strict proviso that no changes or edits are made and the 
original work is properly cited (including links to both the formal publication through the relevant DOI and the license). See: https://creativecommons.org/licenses/by-nc-nd/4.0/.

\section{References}

1. Colagrande S, Inghilesi AL, Aburas S, et al. Challenges of advanced hepatocellular carcinoma. World J Gastroenterol 2016;22:7645-59.

2. Kudo M. A Paradigm Change in the Treatment Strategy for Hepatocellular Carcinoma. Liver Cancer 2020;9:367-77.

3. Finn RS, Qin S, Ikeda M, et al. Atezolizumab plus Bevacizumab in Unresectable Hepatocellular Carcinoma. N Engl J Med 2020;382:1894-905.

4. Llovet JM, Ricci S, Mazzaferro V, et al. Sorafenib in advanced hepatocellular carcinoma. N Engl J Med 2008;359:378-90.

5. Unterrainer M, Ruzicka M, Fabritius MP, et al. PET/ CT imaging for tumour response assessment to immunotherapy: current status and future directions. Eur Radiol Exp 2020;4:63.

6. Kanwal F, Singal AG. Surveillance for Hepatocellular

Cite this article as: Al-Moundhri M, Cubisino A, Panaro F. Hepatocellular carcinoma with complete response to the immunotherapy: the oncologist's dilemma. HepatoBiliary Surg Nutr 2022;11(1):119-122. doi: 10.21037/hbsn-21-499
Carcinoma: Current Best Practice and Future Direction. Gastroenterology 2019;157:54-64.

7. Martins F, Sofiya L, Sykiotis GP, et al. Adverse effects of immune-checkpoint inhibitors: epidemiology, management and surveillance. Nat Rev Clin Oncol 2019;16:563-80.

8. Puzanov I, Diab A, Abdallah K, et al. Managing toxicities associated with immune checkpoint inhibitors: consensus recommendations from the Society for Immunotherapy of Cancer (SITC) Toxicity Management Working Group. J Immunother Cancer 2017;5:95.

9. Vogel A, Cervantes A, Chau I, et al. Hepatocellular carcinoma: ESMO Clinical Practice Guidelines for diagnosis, treatment and follow-up. Ann Oncol 2018;29:iv238-iv255. Erratum in: Ann Oncol 2019;30:871-3.

10. Zhang X, Wang J, Shi J, et al. Cost-effectiveness of Atezolizumab Plus Bevacizumab vs Sorafenib for Patients With Unresectable or Metastatic Hepatocellular Carcinoma. JAMA Netw Open 2021;4:e214846.

11. Couri T, Pillai A. Goals and targets for personalized therapy for HCC. Hepatol Int 2019;13:125-37. 